\title{
INS/GPS Navigation System Based on MEMS Technologies
}

\author{
Vlada Sokolović1, ${ }^{*}$ - Goran Dikić1 ${ }^{-}$Goran Marković ${ }^{2}$ - Rade Stančić1 - Nebojsa Lukić3 \\ 1 University of Defence in Belgrade, Military Academy, Serbia \\ 2 University of Belgrade, School of Electrical Engineering, Serbia \\ ${ }^{3}$ Serbian Armed Forces, Military Test Centre, Serbia
}

Integrated navigation systems are widely used in all aspects of life, i.e. in various civilian and military applications. In the previous two decades, most of the attention in this area has been directed toward the use of low-cost sensors, which are characterized by the low accuracy. Therefore, many algorithms have been developed to implement inertial sensor errors damping, which are based on the integration of inertial sensors with the external sources of information (GPS receivers, magnetometers, barometers, etc.) or mathematical models developed for the self-damping of inertial sensors errors.

This paper proposes a specific method of gyroscope drift compensation by using a PI controller based on the magnetometer measurements, as well as a method of errors compensation in the horizontal channel of navigation system by using the adaptive control signals. The experimental results obtained for the proposed solutions presented in this paper confirmed the possibility of the successful application of these solutions in the real-world environment.

Keywords: barometer, global positioning system, inertial navigation, integration, Kalman filter, magnetometer, MEMS

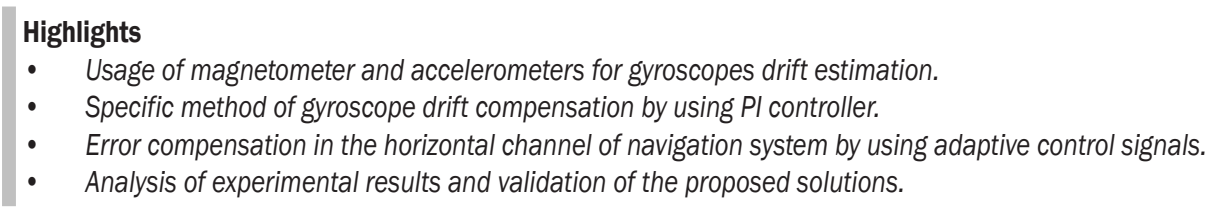

\section{INTRODUCTION}

In accordance with current global trends, specifically the demand to achieve the highest possible accuracy of navigation devices while simultaneously minimizing their costs, low-cost sensors, such as sensors made in micro-electro-mechanical systems (MEMS) technology, have increasing practical application. The largest contribution to the total error of inertial navigation systems (INS) is from the errors of inertial sensors [1]. In comparison to high accuracy sensors, the errors of low-cost sensors have a nonlinear nature that is very difficult to model and to alleviate. Consequently, there is a large error in determining the position and the attitude of the object when these low-cost sensors are used [1]. Bearing in mind that the computation of the new velocity and position values by the navigation algorithm are obtained based on the previous computations, it is obvious that these errors are cumulative and that their values increase rapidly in time, especially for a long-term navigation process. Therefore, it is necessary to perform a periodic correction of the position and velocity values by using an additional navigation system or other independently acquired external measurements [1].

By increasing the accuracy of calculated navigation solution developed by using the low-cost sensors, the construction costs of the INS equipment would decrease. As a result, the relatively weak demand for their usage, which is a key problem in current research in the field of navigation systems based on MEMS technology, should increase.

The Global Positioning System (GPS) is most commonly used as an external source of information for the correction of INS navigation parameters [1]. However, GPS accuracy depends on the spatial arrangement of the GPS satellites relative to the observed receiver, the occurrence of multipath signal propagation, the specific influence of the ionosphere on the GPS signal propagation and the presence of noise in the GPS receiver [2] to [4].

In order to improve the accuracy of the integrated navigation solution, especially for the periods when the GPS receiver does not provide an output with the navigation information, other sensors, such as magnetometers or barometric altimeters (composed of a temperature and a pressure sensor), can be used in addition to the integrated INS/GPS solution. The previously mentioned sensors are also characterized by the specific errors that can be modelled and damped.

In this paper, the results of the research conducted with the goal to develop a model of an integrated INS/GPS/Barometer/Magnetometer navigation system based on a loosely-coupled (LC) integration method is presented. The validity of this research 
lies in a continuous development of methods and algorithms for sensors integration, which are applied in accordance with the advances in low-cost sensor technology, as the one of the main reasons for further research in this field.

The aim of this research was to develop a model that will provide the unified processing of all collected measurements acquired by using the INS, GPS, barometer and magnetometer, in order to obtain more accurate information on the object's spatial position, velocity and attitude. The practical goal of this study was to enable the development of the real-world navigation system and to create the suitable conditions for the further research in the field of multi-sensor integrated navigation.

The applied methods of modelling, which include the dominant application of mathematical models, are used to determine the errors models for individual sensors and to enable successful implementation of the observed navigation system model in the MATLAB environment. The performed analysis has shown the positive contributions of magnetometer usage in order to determine the attitude of the object, as well as of baro-altimeter usage in order to determine the vertical component of velocity and altitude.

Throughout this study, random processes are described as first-order Gauss-Markov or the additive white noise processes. Furthermore, the errors that occur due to quantization, averaging, rounding of the measured values and conversion between different data types are ignored. The accuracy of the navigation solution is established based on the control points (CP) defined for the test trajectory. These referent CPs are precisely determined by the differential GPS.

A verification of the proposed solutions is performed through the experiment on the land vehicle. Therefore, the proposed solutions are tested based on the data collected during this experiment and with the adequate software implemented in MATLAB.

In this work, several results represent the original contribution in the field of research, of which the most important are the following:

- By using the proportional-integral (PI) controller, the algorithm of the magnetometer and gyroscope triad integration has improved the determination of attitude of the object;

- A method for error damping of the horizontal channel of integrated navigation system, based on the adaptive error damping coefficients, is defined;

- The verified results certainly represent a notable improvement in the given field and, therefore, proposed solutions are suitable for practical application in the real-world navigation systems.

In the second section, the attitude determination with gyroscope drift compensation based on magnetometer and accelerometer triad measurements by utilizing the PI controller is described. In the third section, the error compensation in the horizontal and vertical channel of a "strap-down" INS (SINS) is presented. The general scheme of the proposed INS/ GPS/BAR/MAG integration and basic theory of the Kalman filter (KF) with an adaptive control signal, which is used for the integration, is detailed in the fourth section. The fifth section contains a description and the results of the experimental testing of MEMS sensors as well as of the experimental tests that have been realized by using a land vehicle.

Due to the nonlinear nature of the dynamic systems in practice, there is no single solution to the navigation algorithm. Thus, in the present research, several methods of the estimation of states have been applied in the integrated navigation systems.

Bian et al. proposed an adaptive navigation solution, in which a covariance matrix and a matrix of Kalman gains are adapted by the maximum probability function [5]. Stancic and Graovac analysed an INS/ GPS navigation system, in which the integration is realized with the extended KF (EKF) with the control signal [6]. Noureldin et al. [7] used neural network instead of KF for the INS/GPS integration.

The damping of errors in the vertical channel of INS during the outage of GPS information is usually realized with the baro-altimeter, as described by Readdy and Saraswat [8]. In this work, the attenuation of vertical channel errors is performed by the vertical channel error-damping loop with the external source of altitude from GPS or baro-altimeter measurements. Sokolovic et al. [9], used the adaptive EKF control signal for errors damping in the vertical channel of the integrated navigation system.

The magnetometer is the most commonly used device for object attitude determination. For attitude error damping, Madgwick et al. [10] used the negative gradient between the axis of the gyroscopes and the magnetometer. Sokolovic et al. used gain coefficients for gyros drift-damping error compensation [11]. Moreover, the magnetometer was used for the initial attitude determination and for the INS heading angle correction, e.g. in Hao et al. [12], Zhao and Wang [13] and Han and Wang [14]. 


\section{ATTITUDE DETERMINATION}

In this section, the main idea regarding obtaining information about the attitude from two sources is described. The first source is gyroscopes and the second source is a magnetometer and accelerometers as external sensors. Since gyroscope measurements are subjected to certain known errors, the external sensors are used in order to estimate the gyroscope measurement error by utilizing PI controller approach.

If $\omega_{i}$ denotes the components of angular rotation velocity of the body coordinate system relative to the inertial space, then the quaternion innovation at the time $k+1$ is given as [15],

$$
\mathbf{q}_{k+1}=\mathbf{q}_{k}+\frac{1}{2} \mathbf{q}_{k} \cdot \boldsymbol{\omega} T=\mathbf{q}_{k} \Delta \mathbf{q},
$$

where $T$ is a sampling time, and $\Delta \mathbf{q}$ is updating quaternion.

In order to define the horizontal and vertical reference plane by measurements of accelerometers and magnetometers, an objective function is applied here [10] and [16],

$$
f\left(\mathbf{q}_{N}^{B}, \mathbf{d}_{N}\right)=\mathbf{q}_{N}^{B^{*}} \otimes \mathbf{d}_{N} \otimes \mathbf{q}_{N}^{B},
$$

where $\mathbf{d}_{N}$ is some referent vector described in frame $N$, $\mathbf{q}_{N}^{B}$ is quaternion that describes the orientation of the frame $N$ relative to frame $B$ and $\mathbf{q}_{N}^{B^{*}}$ is the conjugate of $\mathbf{q}_{N}^{B}$. In our case, $N$ is the navigation frame and $B$ is the body (sensor) frame. We assumed that the sensor frame and body frame coincided. A complete form of the objective function can be written as:

$$
f\left(\mathbf{q}_{N}^{B}, \mathbf{d}_{N}\right)=\left[\begin{array}{l}
2 d_{x}\left(\frac{1}{2}-q_{3}^{2}-q_{4}^{2}\right)+2 d_{y}\left(q_{1} q_{4}+q_{2} q_{3}\right)+2 d_{z}\left(q_{2} q_{4}-q_{1} q_{3}\right) \\
2 d_{x}\left(q_{2} q_{3}-q_{1} q_{4}\right)+2 d_{y}\left(\frac{1}{2}-q_{2}^{2}-q_{4}^{2}\right)+2 d_{z}\left(q_{1} q_{2}-q_{3} q_{4}\right) \\
2 d_{x}\left(q_{1} q_{3}+q_{2} q_{4}\right)+2 d_{y}\left(q_{3} q_{4}-q_{1} q_{2}\right)+2 d_{z}\left(\frac{1}{2}-q_{2}^{2}-q_{3}^{2}\right)
\end{array}\right],
$$

where $d_{x}, d_{y}$, and $d_{z}$ are elements of the vector $\mathbf{d}_{N}$, and the indexes $(x, y, z)$ denote the axis of the navigation coordinate system. The reference vector of gravity coincides with the vertical axis of the navigation frame, so the reference vector $\mathbf{d}_{N}$ is defined by using the normalized value of the vector of gravity and is given as:

$$
\mathbf{d}_{g, N}=\left[\begin{array}{llll}
0 & 0 & 0 & 1
\end{array}\right] .
$$

Estimated reference directions of gravity, based on Eq. (3) and Eq. (4), can be written as:

$$
\mathbf{v}=\left[\begin{array}{c}
2\left(q_{2} q_{4}-q_{1} q_{3}\right) \\
2\left(q_{1} q_{2}-q_{3} q_{4}\right) \\
q_{1}^{2}-q_{2}^{2}-q_{3}^{2}+q_{4}^{2}
\end{array}\right]
$$

The reference vector of the magnetic field is calculated based on Eq. (6), where $\mathbf{m}$ is the normalized vector of geomagnetic field measured by magnetometer,

$\mathbf{h}_{x, y, z}=2 \mathbf{m}\left[\begin{array}{ccc}0.5-q_{3}^{2}-q_{4}^{2} & q_{2} q_{3}-q_{1} q_{4} & q_{2} q_{4}+q_{1} q_{3} \\ q_{2} q_{3}+q_{1} q_{4} & 0.5-q_{2}^{2}-q_{4}^{2} & q_{3} q_{4}-q_{1} q_{2} \\ q_{2} q_{4}-q_{1} q_{3} & q_{3} q_{4}+q_{1} q_{2} & 0.5-q_{2}^{2}-q_{3}^{2}\end{array}\right]$.

The geomagnetic field can be considered to have components in one horizontal axis and the vertical axis as;

$$
b_{x}=\sqrt{h_{x}^{2}+h_{y}^{2}}, \quad b_{z}=h_{z},
$$

and the reference vector $\mathbf{d}_{N}$ can be defined as,

$$
\mathbf{d}_{m, N}=\left[\begin{array}{llll}
0 & b_{x} & 0 & b_{z}
\end{array}\right] .
$$

Based on Eqs. (3) and (8), the estimated reference directions of magnetic flux are given as:

$$
\mathbf{w}=\left[\begin{array}{c}
2 b_{x}\left(0.5-q_{3}^{2}-q_{4}^{2}\right)+2 b_{z}\left(q_{2} q_{4}-q_{1} q_{3}\right) \\
2 b_{x}\left(q_{2} q_{3}-q_{1} q_{4}\right)+2 b_{z}\left(q_{1} q_{2}+q_{3} q_{4}\right) \\
2 b_{x}\left(q_{1} q_{3}+q_{2} q_{4}\right)+2 b_{z}\left(0.5-q_{2}^{2}-q_{3}^{2}\right)
\end{array}\right] .
$$

The gyroscope measurement error or the deviation of the measurement vector from the reference vectors is calculated as,

$$
\mathbf{e}=(\mathbf{v} \times) \mathbf{a}+(\mathbf{w} \times) \mathbf{m},
$$

where $(\mathbf{v} \times)$ and $(\mathbf{w} \times)$ denote the skew-symmetric matrices of gravity, and magnetic flux estimated directions and $\mathbf{a}$ is the normalized vector of acceleration.

The gyroscope drift and noise corrections are performed based on the estimated value of the error in the current iteration:

$$
\hat{\omega}_{c}=\omega+\mathrm{K}_{\mathrm{p}} \cdot \mathbf{e}+\mathrm{K}_{\mathrm{i}} \cdot \mathbf{e}_{\mathrm{int}},
$$

where $\boldsymbol{\omega}$ denote the measurements of gyroscopes, $\hat{\boldsymbol{\omega}}_{c}$ denote the estimated (corrected) values of gyroscopes measurements, $\mathrm{K}_{\mathrm{p}}$ is the gyro drift damping coefficient, $K_{i}$ is the gyro measurement noise damping coefficient, and $\mathbf{e}_{\text {int }}$ is the integral of error in current iteration. The coefficients $K_{p}=0.01$, and $K_{i}=0.005$ represent the coefficients of the PI controller and are determined based on the Gauss-Smith method. The PI controller is chosen in order to allow the removal of 
coarse gyroscope drift and to enable the faster response to the sudden appearance of measurements error independently on the previous measurements. Corrected values of gyroscopes measurements are used to determine the updating quaternion and are based on Eq. (1) to calculate quaternion in the current iteration. Based on these values, the final quaternion, transformation matrix $\mathbf{C}_{B}^{P}$, is formed. Index $P$ represents the platform frame. The platform frame is an image of the navigation frame, which is established by an on-board computer. For the ideal sensors, the platform frame coincides with navigation frame $N$. In this paper, a north-east-down (NED) frame is used as a navigation frame. The fine filtering is realized by the EKF, which performs the estimation of the attitude errors that are used to form the matrix $\mathbf{C}_{P}^{N}$. Thus, the final solution of the attitude is determined by the expression:

$$
\mathbf{C}_{B}^{N}=\mathbf{C}_{P}^{N} \mathbf{C}_{B}^{P},
$$

where $\mathbf{C}_{B}^{N}$ is the resultant matrix, whose elements are used to determine the attitude of the object body relative to the navigation coordinate system, i.e. roll, pitch and yaw angles, marked as $\phi, \theta, \psi$. The functional diagram of the strap-down INS, with the coordinate systems that are used in this work, is shown in Fig. 1.

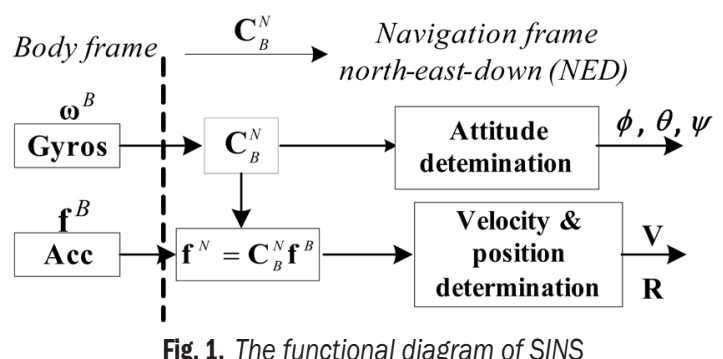

In Fig. 1, $\boldsymbol{\omega}^{B}$ denotes gyroscopes measurements in the body frame and $\mathbf{f}^{B}$ denotes specific forces, measured by the accelerometers, in the body frame.

\section{INS ERRORS DAMPING}

The proposed solution, specifically the error compensation for the horizontal and vertical channels of INS, is achieved with the introduction of external information, e.g. the additional measurements obtained with the other sensors. In this section, the algorithm for errors damping for the horizontal and vertical channels of INS is presented. The single channel error diagram for INS with error damping by introducing external information from GPS is shown in Fig. 2. In this figure $\mathrm{K}_{1}$ and $\mathrm{K}_{2}$ are coefficients, $\Phi_{e}$ is attitude error in east direction (difference between gyro platform and navigation frames), $\Phi_{e}(0)$ is initial misalignment error between platform and navigation frames, $\delta V_{n}$ is velocity error, $R e$ is the radius of the Earth, $\delta R_{n}$ is position error, $\delta a B_{n}$ are accelerometers "bias" and $\delta g B_{e}$ is gyro drift.

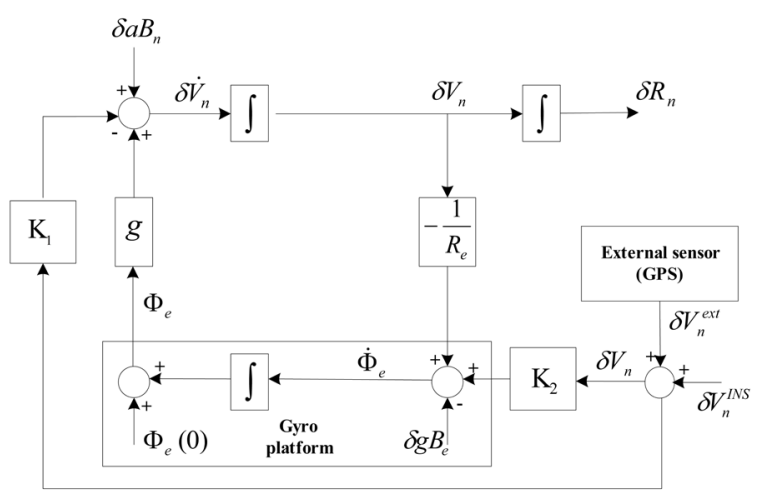

Fig. 2. The North Channel error diagram of INS, with error damping by the GPS measurements usage

Based on the diagram in Fig. 2, the single channel error model is defined as:

$$
\begin{aligned}
& \delta \dot{V}_{n}=g \Phi_{e}-K_{l} \delta V_{n}+\delta a B_{n}, \\
& \dot{\Phi}_{e}=-\frac{\delta V_{n}}{R_{e}}-K_{2} \delta V_{n}+\delta g B_{e} .
\end{aligned}
$$

By obtaining the derivatives of the first equation in Eq. (13) (derivatives of $\delta \dot{V}_{n}$ ), and introducing the second equation of Eq. (13) into the first equation of Eq. (13), we obtain:

$$
\delta \ddot{V}_{n}+\mathrm{K}_{1} \delta \dot{V}_{n}+\left(\omega_{s}^{2}+\mathrm{K}_{2} g\right) \delta V_{n}=-g \cdot \delta g B_{e}+\delta a \dot{B}_{n} \cdot(14
$$

The left side of Eq. (14) describes a second-order oscillator. The selection of optimal coefficients $K_{1}$ and $\mathrm{K}_{2}$ is based on a compromise between the value of the static error and the system bandwidth. A selection of high values of $K_{1}$ and $K_{2}$ provides a low static error value, but in this case, the system is characterized by a wide bandwidth in order to allow transmission of high-frequency noise components [14]. Therefore, the selection of optimal coefficients, $\mathrm{K}_{1}$ and $\mathrm{K}_{2}$, should be performed as a compromise between these two opposite requirements. As the MEMS sensors inherently produce high static errors, the values of $K_{1}$ and $K_{2}$, can be very high. Such behaviour may cause the instability of the EKF; therefore, we have to separate these coefficients. The first pair of coefficients is marked as $\mathrm{K}_{1 \mathrm{INS}}$ and $\mathrm{K}_{2 \mathrm{INS}}$ and are used for attenuation of INS errors, while the second pair of 
coefficients, $\mathrm{K}_{1 \mathrm{KF}}$ and $\mathrm{K}_{2 \mathrm{KF}}$, are used in the EKF in order to form control signals, as given in:

$u_{n}^{v}=-\mathrm{K}_{1 n K F} \sinh \left(\delta \hat{V}_{n}\right), \quad u_{e}^{v}=-\mathrm{K}_{1 e K F} \sinh \left(\delta \hat{V}_{e}\right)$,

$u_{n}^{\varphi}=-\mathrm{K}_{2 n K F} \sinh \left(\delta \hat{V}_{e}\right), \quad u_{e}^{\varphi}=-\mathrm{K}_{2 n K F} \sinh \left(\delta \hat{V}_{n}\right)$,

where $\mathrm{K}_{1 n K F}=\mathrm{K}_{1 e K F}=0.01$, and $\mathrm{K}_{2 n K F}=\mathrm{K}_{2 e K F}=$ 0.007 .

The control signals at the horizontal channel, which are shown in Fig. 3, are defined by using the maximum expected velocity difference $\delta \widehat{V}_{n}$ (in our case $1.1 \mathrm{~m} / \mathrm{s}$ ), i.e. $\delta \hat{V}_{e}$ for the eastern channel.

During the period of GPS data outage, the control signals are formed on the basis of the moving average values computed for the last 16 measurements of velocity, $V_{n}$ and $V_{e}$, in order to obtain average values $V_{n m a}$ and $V_{e m a}$.
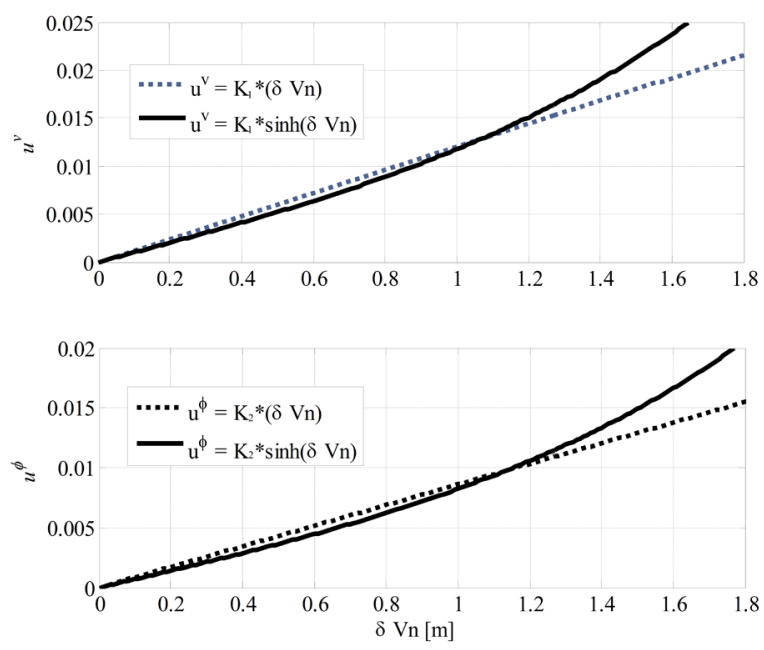

Fig. 3. The control signals at the horizontal channel of the proposed INS solution

In existing solutions [6] and [11], these values were used for the auto-correction of the horizontal channel errors, while in the solution proposed here, these values are used in order to compute the velocity vector of movement in the horizontal plane $V_{\text {Hma }}=\sqrt{V_{n m a}^{2}-V_{\text {ema }}^{2}}$, which can be decomposed into the north and east components of velocity by using the yaw angle. This way, we can determine:

$$
\begin{gathered}
\delta \hat{V}_{n}^{I N S}=V_{n I N S}-V_{H m a} \cos (\psi), \text { and } \\
\delta \hat{V}_{e}^{I N S}=V_{e I N S}-V_{H m a} \sin (\psi) .
\end{gathered}
$$

In order to achieve more effective error damping in case of GPS data outage, the higher values of coefficients $\mathrm{K}$ are necessary. For this reason the adaptation of these coefficients by using the nonlinear hyperbolic functions $\sinh ()$ is performed and the values of coefficients $\mathrm{K}$ are selected to reach the maximum expected value of $\delta V_{n, e}$. The function $\sinh ()$ is defined for a wide domain of input values, with the property that as the input signal error values rise the function response increases slowly for small inputs (almost with a linear rise), while it increases more quickly for the higher signal error values.

The adaptation of the coefficients in the vertical channel is adopted as described in [9], and the model of the vertical channel is given as:

$$
\begin{gathered}
\delta \dot{h}=\delta V_{z}-C_{1} \sinh \left(\delta h-\delta h^{B A R}\right), \\
\delta \dot{V}_{z}=\delta A_{z}-C_{2} \sinh \left(\delta h-\delta h^{B A R}\right)-\delta a+2 \omega_{s}^{2} \delta h,
\end{gathered}
$$

where $\delta V_{z}$ is the velocity error, $\delta h$ is the INS height error, $\delta A_{z}$ is the vertical acceleration error, $\delta h^{B A R}$ is the baro-altimeter error, $\delta a$ is the steady state error, $\omega_{s}$ is Shuler's frequency, and $\mathrm{C}_{1}, \mathrm{C}_{2}$ are control coefficients pair for vertical channel error. The high values of these coefficients can cause system instability and, therefore, the separation of these coefficients is introduced, with $\mathrm{C}_{1 \mathrm{INS}}, \mathrm{C}_{2 \mathrm{INS}}$ being coefficients for the INS correction, and $\mathrm{C}_{1 \mathrm{KF}}, \mathrm{C}_{2 \mathrm{KF}}$, being coefficients for the control signals in EKF. The coefficient values that were used in this paper are $\mathrm{C}_{1 \mathrm{INS}}=0.04, \mathrm{C}_{2 \mathrm{INS}}=0.8$, $\mathrm{C}_{1 \mathrm{KF}}=0.035$ and $\mathrm{C}_{2 \mathrm{KF}}=3.2 \times 10^{-4}$.

When there are no valid GPS measurement data, the EKF works in the prediction mode, based on the covariance matrices $\mathbf{Q}$ (system noise) and $\mathbf{R}$ (measurement noise).

\section{INTEGRATED NAVIGATION SYSTEM}

The block scheme of the INS/GPS/BAR/MAG integration is shown in Fig. 4. In accordance with a solution for INS errors damping, the errors model, used in the presented integrated scheme for INS errors estimation, is given in [1].

The variables given in Fig. 4 are defined as: $\delta V_{n}, \delta V_{e}$, $\delta V_{d}$ are linear velocity errors NED components, $\delta \varphi$, $\delta \lambda, \delta h$ are position errors components, $\phi_{n}, \phi_{e}, \phi_{d}$ present orientation errors NED components for calculated platform, $\omega_{n}^{d r}, \omega_{e}^{d r}, \omega_{d}^{d r}$ are gyro drifts, $\mathbf{w}(t)$ is Gaussian white noise, while $B_{n}, B_{e}, B_{d}$ present NED components of the accelerometer biases.

Based on the estimation error model, the correction of the velocity components in the NED coordinate system, is defined as:

$$
\begin{aligned}
V_{n}^{c} & =V_{n}^{I N S}-\delta \widehat{V}_{n}, V_{e}^{c}= \\
& =V_{e}^{I N S}-\delta V_{e}^{\rightleftarrows}, V_{d}^{c}=V_{d}^{I N S}-\delta \widehat{V}_{d},
\end{aligned}
$$


where $\delta \widehat{V}_{n}, \delta \widehat{V}_{e}, \delta \hat{V}_{d}$ are estimates of velocity errors defined at the output of EKF. The position correction is given as:

$$
\varphi^{c}=\varphi^{I N S}-\delta \hat{\varphi}, \lambda^{c}=\lambda^{I N S}-\delta \hat{\lambda}, h^{c}=h^{I N S}-\delta \hat{h},
$$

where $\delta \hat{\varphi}, \delta \hat{\lambda}, \delta \hat{h}$ are estimates of position errors for the LLh (latitude, longitude, and height) coordinates.

The state vector in EKF, in our case, is given as, $\mathbf{x}=\left[\begin{array}{lllllllll}\delta r_{\phi} & \delta r_{\lambda} & \delta r_{h} & \delta V_{n} & \delta V_{e} & \delta V_{d} & \delta \varphi & \delta \theta & \delta \psi\end{array}\right.$

$$
\left.\begin{array}{llllll}
B_{n} & B_{e} & B_{d} & \omega_{n}^{d r} & \omega_{e}^{d r} & \omega_{d}^{d r}
\end{array}\right] .
$$

The states in the EKF with control signals can be represented as:

$$
\hat{\mathbf{x}}_{k}^{+}=\Phi_{k} \hat{\mathbf{x}}_{k-1}^{+}+\mathbf{u}_{k}+\mathbf{K}_{k}\left(\mathbf{z}_{k}-\mathbf{H}_{k} \boldsymbol{\Phi}_{k} \hat{\mathbf{x}}_{k-1}^{+}-\mathbf{H}_{k} \mathbf{u}_{k}\right)
$$

where $\mathbf{u}$ is matrix defined as:

$$
\mathbf{u}_{k-1}=\operatorname{diag}\left(\left[\begin{array}{lllllllll}
0 & 0 & u_{h}^{r} & u_{n}^{v} & u_{e}^{v} & u_{d}^{v} & u_{n}^{\varphi} & u_{e}^{\varphi} & 0
\end{array}\right]\right) \text {, }
$$

while $\mathbf{K}_{k}$ is the Kalman gain matrix, and $\boldsymbol{\Phi}$ is the state transition matrix. The control signals are given as:

$$
\begin{aligned}
& u_{n}^{v}=-K_{I n K F} \sinh \left(\delta \hat{V}_{n}\right), u_{e}^{v}=-K_{\text {leKF}} \sinh \left(\delta \hat{V}_{e}\right), \\
& u_{n}^{\varphi}=-K_{2 n K F} \sinh \left(\delta \hat{V}_{e}\right), u_{e}^{\varphi}=-K_{2 e K F} \sinh \left(\delta \hat{V}_{n}\right), \\
& u_{h}^{r}=-C_{I K F} \sinh (\delta h), u_{d}^{v}=-C_{2 K F} \sinh (\delta h) .
\end{aligned}
$$

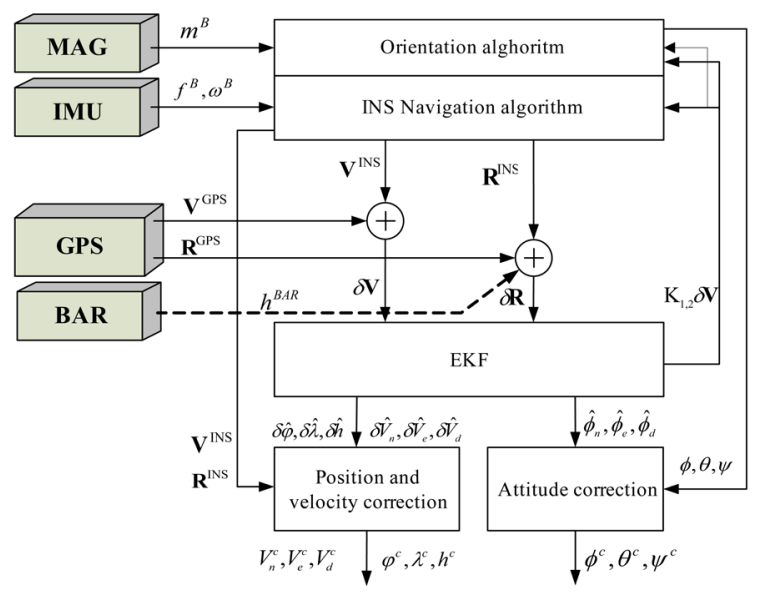

Fig. 4. Block scheme of the observed INS/GPS/BAR/MAG integration

The Kalman gains are time varying, with the steady state defined for the equilibrium between the process noise variance accumulation and the measurement noise variance. These steady-state gains in the Kalman filter are the same as the optimum $\mathrm{K}$ and $\mathrm{C}$ coefficients used for error damping in the INS. It should be noted that the adaptation of these coefficients is performed only when GPS data are not available.
In the case of GPS drop-out, EKF is used to perform prediction with the Kalman gain matrix formed as zero matrix $(\mathbf{K}=0)$.

\section{EXPERIMENTAL RESULTS}

The integrated navigation system used in this study is formed by using the low cost INS MEMS sensors: the MPU-60X0, which combines a 3-axis gyroscope and a 3-axis accelerometer, "Gms-u1LP" GPS receiver (L1 C/A code, updating frequency $10 \mathrm{~Hz}$ ) and the MS5611-01BA03 as a barometric pressure sensor.

We assumed the non-orthogonality of the INS sensors as defined in product specifications. The stochastic noise characteristic of these sensors was determined by the Allan variance method based on a three-hour measurements. These data are used for the sensor calibration and for the covariance matrices used in EKF initialization. The results of those tests are given in Table 1.

Experimental testing of the integrated navigation system was performed by using a land vehicle. The device was installed on the center of mass of the vehicle and the antenna of the GPS receiver was placed on the top of the vehicle, wherein the applied software

\begin{tabular}{|c|c|c|c|}
\hline Acc & $\begin{array}{c}\text { Bias } \\
{\left[\mathrm{m} / \mathrm{s}^{2}\right]}\end{array}$ & $\begin{array}{c}\text { White noise } \\
{\left[\mathrm{m} / \mathrm{s} / \mathrm{s}^{1 / 2}\right]}\end{array}$ & $\begin{array}{c}\text { Random walk } \\
{\left[\mathrm{m} / \mathrm{s}^{2} / \mathrm{s}^{1 / 2}\right]}\end{array}$ \\
\hline$x$ & $-11.5 \times 10^{-2}$ & $1.77 \times 10^{-2}$ & $6.48 \times 10^{-4}$ \\
\hline$y$ & $-1.66 \times 10^{-2}$ & $1.86 \times 10^{-2}$ & $5.73 \times 10^{-4}$ \\
\hline$z$ & $9.8 \times 10^{-1}$ & $1.87 \times 10^{-2}$ & $1.94 \times 10^{-3}$ \\
\hline Gyro & $\begin{array}{c}\text { Drift } \\
{[\mathrm{rad} / \mathrm{s}]}\end{array}$ & $\begin{array}{l}\text { White noise } \\
{\left[\mathrm{m} / \mathrm{s} / \mathrm{s}^{1 / 2}\right]}\end{array}$ & $\begin{array}{l}\text { Random walk } \\
{\left[\mathrm{m} / \mathrm{s}^{2} / \mathrm{s}^{1 / 2}\right]}\end{array}$ \\
\hline$x$ & $9.28 \times 10^{-3}$ & $11.3 \times 10^{-3}$ & $3.7 \times 10^{-4}$ \\
\hline$y$ & $2.8 \times 10^{-3}$ & $10.5 \times 10^{-3}$ & $3.04 \times 10^{-4}$ \\
\hline$z$ & $1.02 \times 10^{-4}$ & $10.2 \times 10^{-3}$ & $6.07 \times 10^{-5}$ \\
\hline \multirow[t]{2}{*}{ Baro } & $\tau_{\mathrm{c}}[\mathrm{s}]$ & $\begin{array}{l}\text { White noise } \\
{\left[\mathrm{m} / \mathrm{s}^{1} \mathrm{~s}^{1 / 2}\right]}\end{array}$ & $\begin{array}{l}\text { Random walk } \\
{\left[\mathrm{m} / \mathrm{s}^{2} / \mathrm{s}^{1 / 2}\right]}\end{array}$ \\
\hline & 200 & $9.31 \times 10^{-3}$ & $13.9 \times 10^{-2}$ \\
\hline
\end{tabular}
solution was used to adapt to the position differences between the GPS antenna and the inertial sensors. The vehicle was moving along the predetermined trajectory, in the urban environment, which contains ups and downs, and which is defined with twenty CP.

Table 1. Sensors error parameterization

The covariance matrix $\mathbf{R}$, is formed based on the 12 hours long GPS measurements at the known position and is given as: $R_{1,1}=5.7296 \mathrm{e}-8 ; R_{2,2}=$ $4.9 \mathrm{e}-8 ; \mathrm{R}_{3,3}=1.55 ; \mathrm{R}_{4,4}=0.01 ; \mathrm{R}_{5,5}=0.01 ; \mathrm{R}_{6,6}=$ 0.01 . Covariance matrix $\mathbf{Q}$ is determined based on the sensors' noise characteristics and is given as: $\mathrm{Q}_{3,3}$ $=1 \mathrm{e}-4 ; \mathrm{Q}_{4,4}=4.77 \mathrm{e}-5 ; \mathrm{Q}_{5,5}=2.75 \mathrm{e}-6 ; \mathrm{Q}_{6,6}=7.3 \mathrm{e}-3$; 
$\mathrm{Q}_{7,7}=1.5 \mathrm{e}-3 ; \mathrm{Q}_{8,8}=1.5 \mathrm{e}-3 ; \mathrm{Q}_{9,9}=1 \mathrm{e}-3 ; \mathrm{Q}_{10,10}=1.2 \mathrm{e}-$ $3 ; \mathrm{Q}_{11,11}=1.2 \mathrm{e}-3 ; \mathrm{Q}_{12,12}=1 \mathrm{e}-4 ; \mathrm{Q}_{13,13}=1 \mathrm{e}-4 ; \mathrm{Q}_{14,14}=$ 1e-4; $Q_{15,15}=1 \mathrm{e}-4$; The other elements of the matrices $\mathbf{R}$ and $\mathbf{Q}$ are zero.

The paper presents the test results obtained for a period of 300 seconds. All system settings were set in stationary conditions. In Fig. 5, the accelerometers' row data acquired during the test are shown, as well as the results after filtering performed based on the parameters of the stochastic sensors.

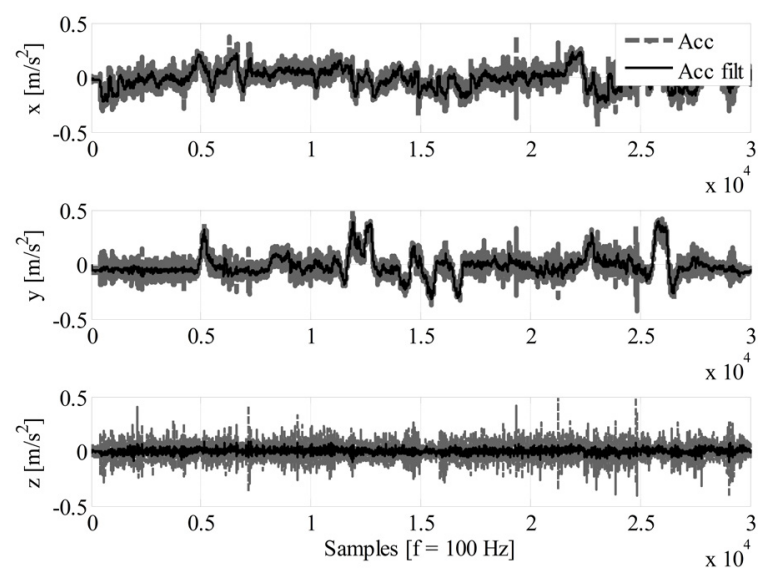

Fig. 5. Accelerometers measurements obtained during the test

In Fig. 6, the results of the gyroscopes measurements, acquired during the test are shown, with the results of these measurements after filtration. In these figures, in both cases, the large influence of noise is evident, especially for the low values of the sensors measurements. This fact is particularly noticeable on the vertical axis of gyroscopes triad in Fig. 6.
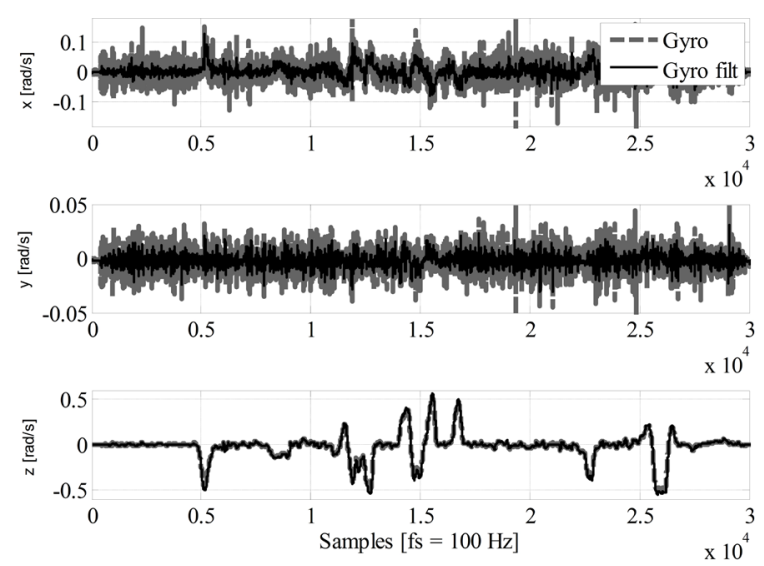

Fig. 6. Gyroscope measurements obtained during the test

The normalized values of the magnetometer measurements after calibration, in the horizontal plane, are shown in Fig. 7. As the graph describes a full circle, it is clear that the hard iron measurement errors are removed when the calibration method is applied. Deviations of the graph from the exact circle are a consequence of the soft iron errors, which have not been entirely removed.

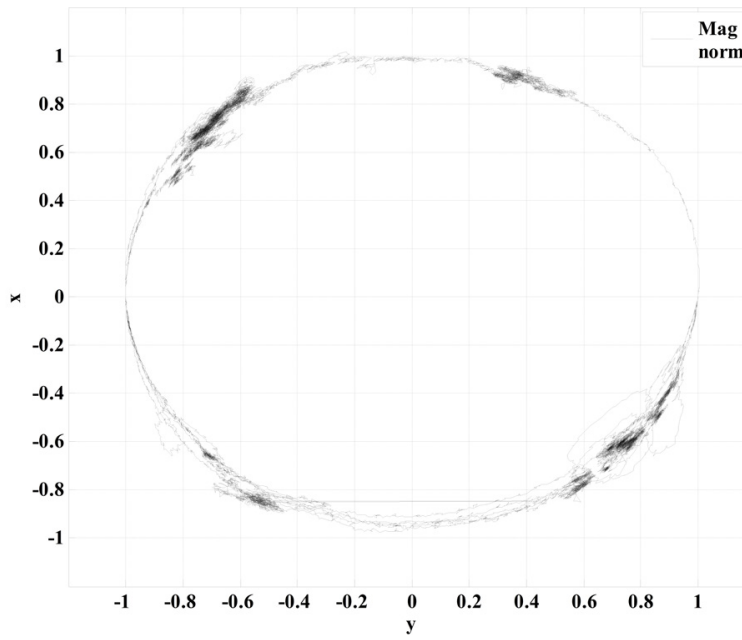

Fig. 7. Normalized magnetometer measurements in the horizontal plane, after the calibration, acquired during the test

The results of pressure and temperature sensor measurements during the test are shown in Fig. 8. In this figure, the black lines represent the filtered measurements. Bearing in mind the noise characteristics of the pressure sensor, shown in Table 1 , there is no obvious strong presence of noise in Fig. 8 , because the dominant barometer error is random walk noise. The step graph of temperature row data, shown in the lower graph in Fig. 8, is a consequence of sensor resolution of $0.1{ }^{\circ} \mathrm{C}$.
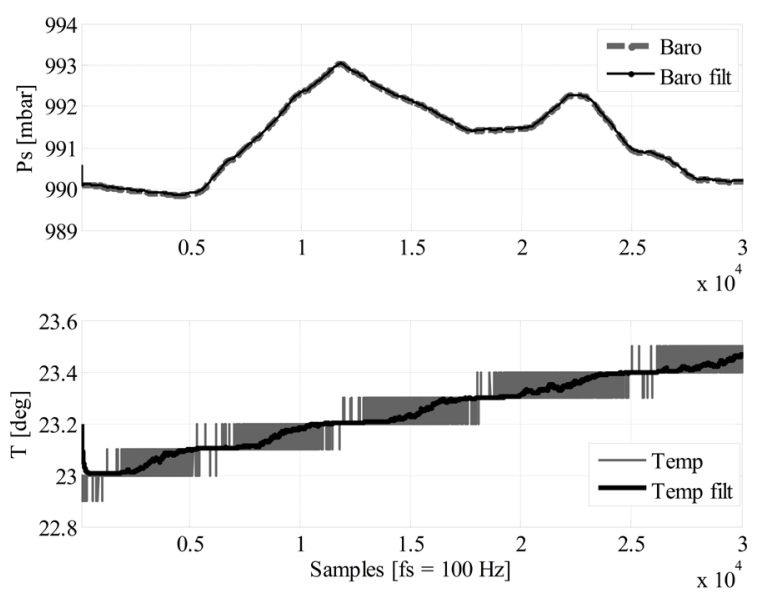

Fig. 8. Measurements of the barometer and temperature sensors, before and after filtration 
A profile of the trajectory along which the test was performed is shown in Fig. 9. The CPs are selected in such a way to allow full control of determining the position of the vehicle during its movement, e.g. at critical points on the road, such as the changes of ups and downs and curves.

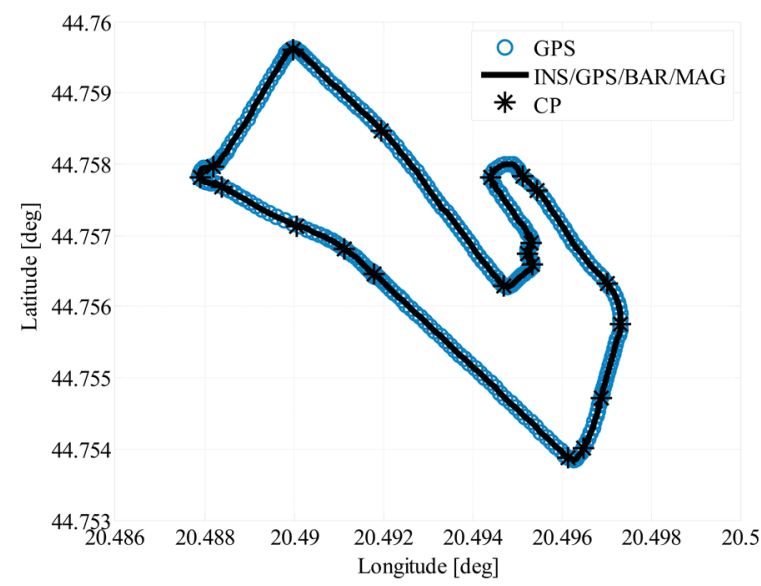

Fig. 9. The trajectory of vehicle movement in the horizontal plane, with marked control points

Horizontal and vertical profile of vehicle trajectory in NED coordinates, relative to the GPS measurements are given in Figs. 10 and 11. During these tests, there was no interruption of the GPS information. The vertical profile of the trajectory is particularly interesting because of the large deviations of GPS measurements relative to the CPs, which resulted with the large covariance measurement error of the altitude.
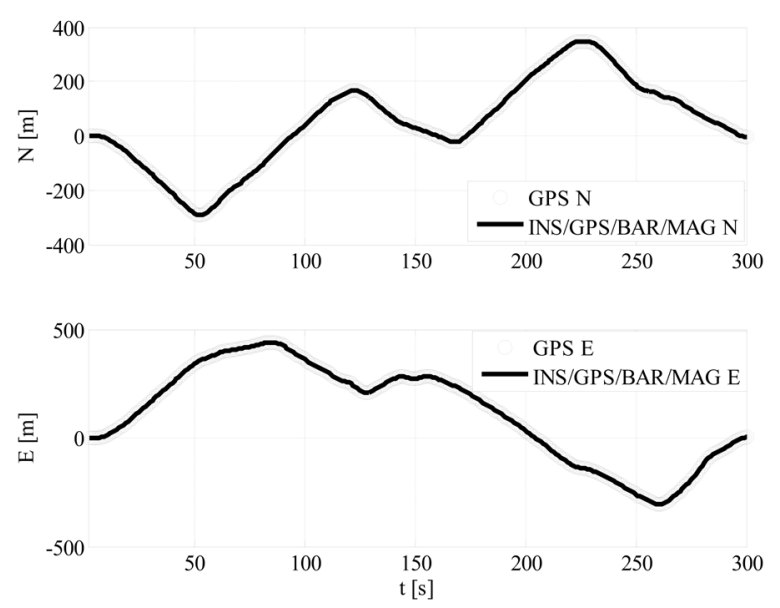

Fig. 10. The north and east coordinates, of the vehicle trajectory in NED coordinate system (when GPS is always on)

The vehicle velocity in the NED coordinates is shown in Fig. 12. As the updating frequency of GPS data is $10 \mathrm{~Hz}$, the likelihood of a large navigation error accumulation between the GPS measurements is very low (negligible). Furthermore, with the well-defined EKF parameters, the measurement noise is removed, as it is evident in Fig. 12.

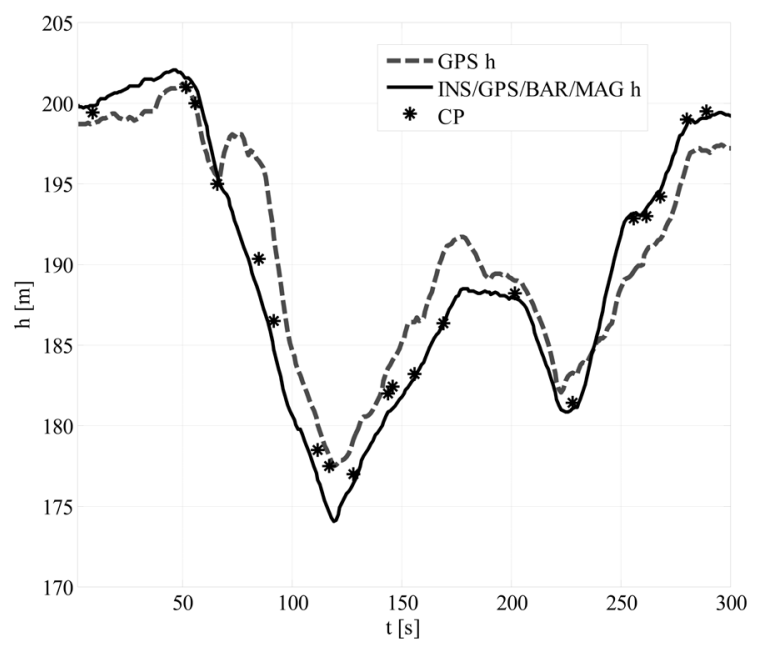

Fig. 11. The altitude profile of vehicle trajectory, (when GPS is always on)

In the upper graph in Fig. 13, the heading angle of the vehicle is shown. The angle was determined based on full integration of gyros, accelerometers, and the magnetometer, where the angle change is in accordance with the trajectory of the vehicle. The deviation of yaw angle for different cases of sensor integration is shown in the lower graph in Fig. 8. All these deviations are reduced to a small value by the use of additional external measurements, especially those of magnetometer.
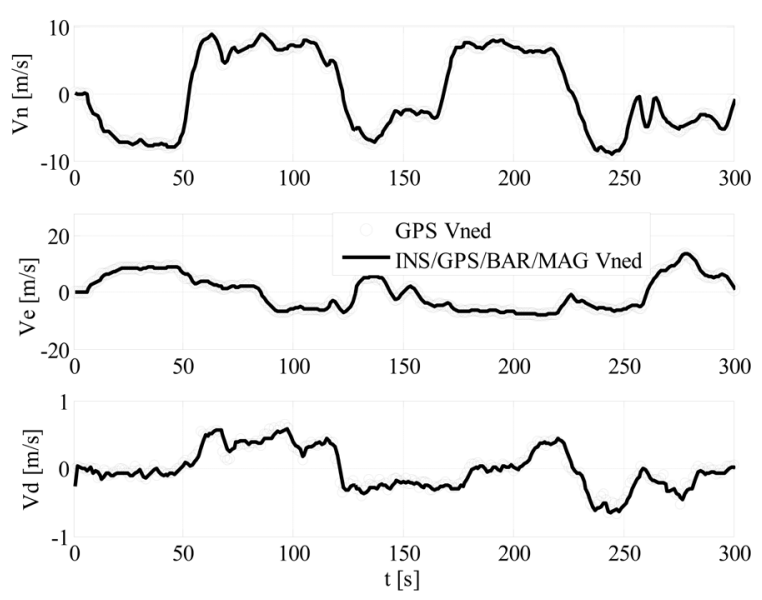

Fig. 12. Velocity profiles of the vehicle in NED coordinate system, (GPS on) 
The particularly large positive contribution of gyroscopes and magnetometers integration can be seen through the determination of the pitch angle, as given in Fig. 14. When observing the results of measurements relative to the specific angles defined with CPs, it is clear that the best results are obtained when the gyroscopes drift suppression is done by using the magnetometers and accelerometers. The CPs can only approximately determine the pitch angle; therefore, it is not possible to accurately determine the measurement error.
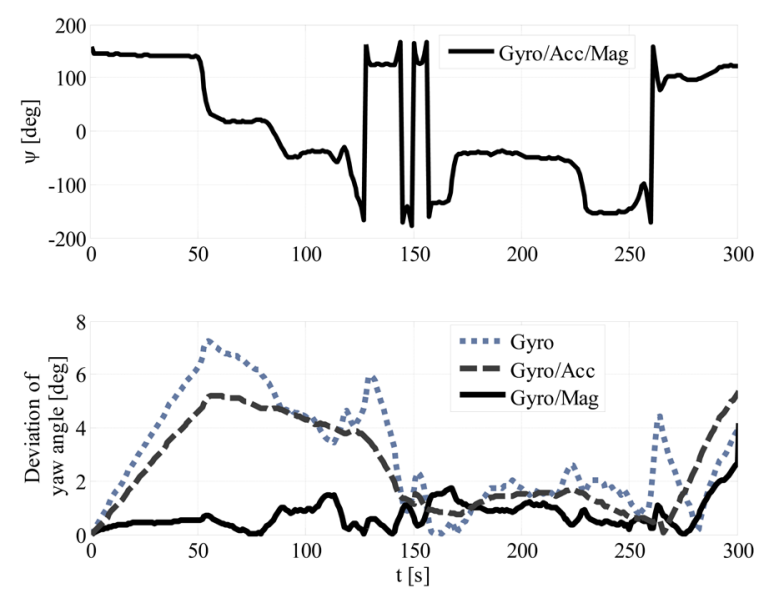

Fig. 13. The flow of the yaw angle during the experimental testing

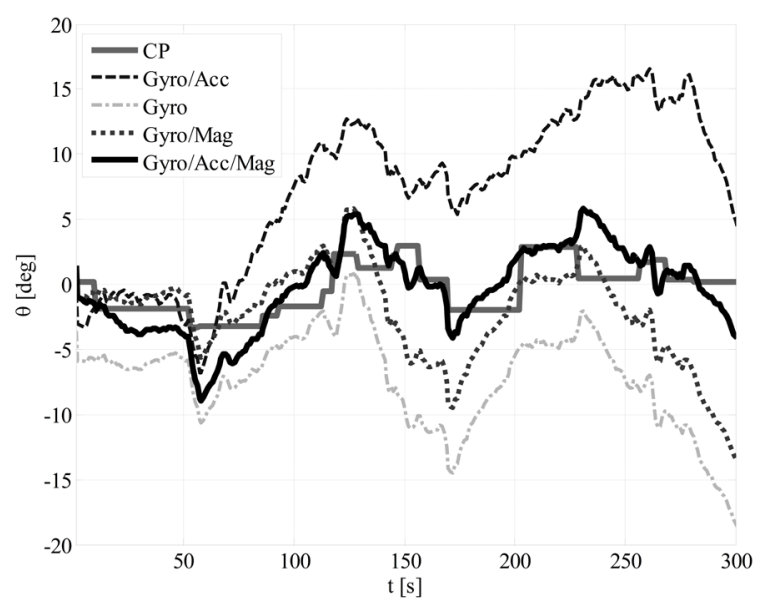

Fig. 14. The flow of the pitch angle during the experimental testing

The improvement of the roll angle accuracy is evident in Fig. 15, which shows the transition of the roll angle along the experimental trajectory. By observing the roll and pitch angle curves, it is evident that there are large divergences in cases in which the angles are determined only based on the gyroscope measurements, which is the consequence of the gyro sensors imperfections. A partial gyroscope drift compensation is achieved by the introduction of accelerometer measurements into the orientation algorithm.

However, accelerometers do not produce perfect data and therefore gyroscopes drift cannot be absolutely removed. The largest deviations of the pitch and roll angles occur when there are changes of direction and velocity of the vehicle, which is in accordance with Figs. 10 to 12 . The best possible results are achieved with the integration of all three sensor types.

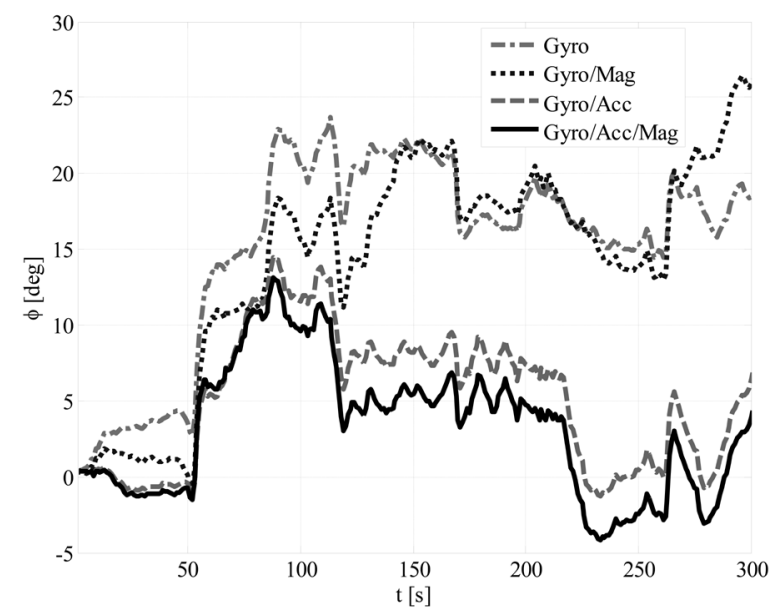

Fig. 15. The flow of the roll angle during the experimental testing

The graphics of the vehicle position errors in the horizontal and vertical plane for the period of GPS information absence (artificially introduced between $180 \mathrm{~s}$ to $210 \mathrm{~s}$ ) for a period of 30 seconds are shown in Fig. 16. The self-damping algorithm of INS measurements is realized as described in Section 3. The rapid increasing of the position error is typical for the MEMS sensors.

The curves in Figure 16 show the case when there are linear acceleration of the vehicle, in all three directions, during the manoeuvre. The maximum, mean and root mean square error (rms) of measurements are shown in Tab. 2. The case in which there are linear and angular accelerations of the vehicle, during the GPS outage, exist in a period between $150 \mathrm{~s}$ and $160 \mathrm{~s}$. In this case, there is a large error in determination the position and velocity of the vehicle. The errors of the position and velocity in this case are also shown in Table 2.

For comprehensive and unequivocal monitoring of navigation parameters, the velocity deviations, during the GPS data outages, are also displayed for the time interval between $180 \mathrm{~s}$ and $210 \mathrm{~s}$ in Fig. 17. 
The errors of vehicle velocity are also shown in Table 2.

If we look at the graphics in Figs. 16 and 17, and the results that are shown in Table 2, it can be concluded that the proposed solutions for the object attitude, position and velocity errors damping allow the successful realization of the real multi-sensor integrated navigation system, which is based on MEMS sensors.
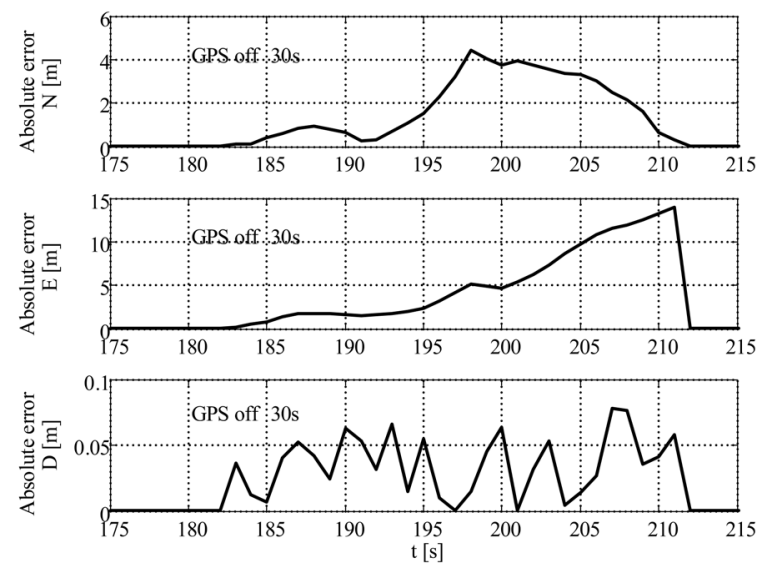

Fig. 16. Vehicle position errors during the GPS outage
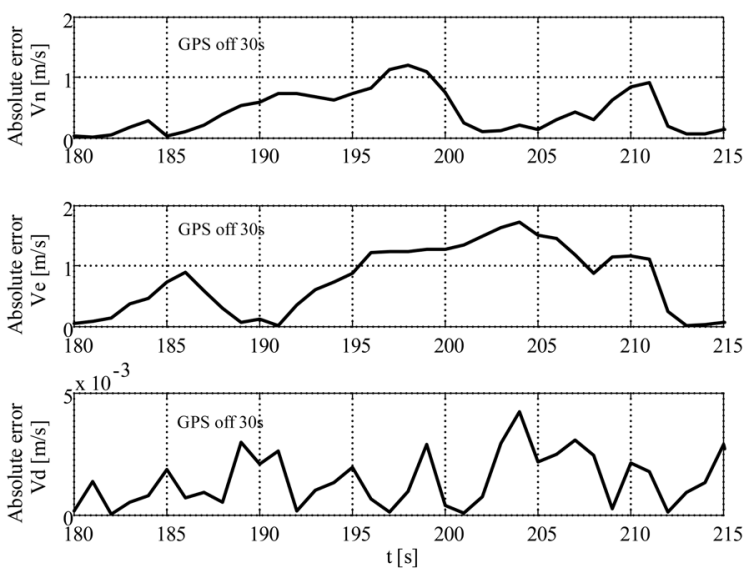

Fig. 17. The velocity estimation in NED coordinates, during the GPS outage

Table 2. Summary of the experimental results

\begin{tabular}{lcccccc}
\hline \multirow{2}{*}{ Parameter } & \multicolumn{3}{c}{ Error (GPS off 10 s) } & \multicolumn{3}{c}{ Error (GPS off 30 s) } \\
\cline { 2 - 7 } & $\max$ & mean & rms & $\max$ & mean & rms \\
\hline $\mathrm{N}[\mathrm{m}]$ & 8.209 & 4.021 & 4.809 & 5.141 & 1.801 & 2.320 \\
\hline $\mathrm{E}[\mathrm{m}]$ & 9.301 & 2.231 & 3.491 & 13.894 & 4.840 & 6.430 \\
\hline $\mathrm{D}[\mathrm{m}]$ & 0.071 & 0.024 & 0.029 & 0.109 & 0.035 & 0.042 \\
\hline $\mathrm{Vn}[\mathrm{m} / \mathrm{s}]$ & 1.158 & 0.042 & 0.746 & 1.231 & 0.497 & 0.602 \\
\hline $\mathrm{Ve}[\mathrm{m} / \mathrm{s}]$ & 1.561 & 0.031 & 0.823 & 1.759 & 0.882 & 1 \\
\hline $\mathrm{Vd}[\mathrm{m} / \mathrm{s}]$ & 0.123 & 0.009 & 0.002 & 0.135 & 0.009 & 0.019 \\
\hline
\end{tabular}

\section{CONCLUSIONS}

The results of the comprehensive analysis, observed in the accordance with the objectives of the research, clearly show that the proposed solutions for the error damping justify the initial assumptions and objectives of the conducted research. It can be also concluded that the usage of the magnetometer for gyro drift compensation through the PI controller usage contributes to the improvement in the attitude determination of the vehicle. Finally, we have shown that the proposed solution for the self-error damping in the horizontal channel produces reliable results in the user position and velocity determination process, although only the low-cost sensors are used.

\section{ACKNOWLEDGEMENT}

This work was done within the research project of the Ministry of Science and Technological Development of Serbia III47029.

\section{REFERENCES}

[1] Salychev, 0.S. (2012). MEMS-based Inertial Navigation: Expectations and Reality. The Bauman Moscow State Technical University, Moscow

[2] Sokolović, V. (2011). Analyse of signal acquisition in GPS software receiver. Vojnotehnički glasnik / Military Technical Courier, vol. 59, no. 1, p. 81-95. (in Serbian)

[3] Kuščer, L., Diaci, J. (2013). Measurement Uncertainty Assessment in Remote Object Geolocation. Strojniški vestnik - Journal of Mechanical Engineering, vol. 59, no. 1, p. 32-40, DOI:10.5545/sv-jme.2012.642.

[4] Gülal, E., Dindar, A.A., Akpınar, B., Tiryakioğlu, I., Aykut, N.O., Erdoğan, H. (2015). Analysis and management of GNSS reference station data. Tehnični vjestnik - Technical Gazette, vol. 22, no. 2, p. 407-414, Dol:10.17559/tv-20140717125413.

[5] Bian, H., Jin, Z., Tian, W. (2006). IAE-adaptive Kalman filter for INS/GPS integrated navigation system. Journal of Systems Engineering and Electronics, vol. 17, no. 3, p. 502- 508, DOI:10.1016/S1004-4132(06)60086-8.

[6] Stancic, R., Graovac, S. (2010). The integration of strap-down INS and GPS based on adaptive error damping. Robotics and Autonomous Systems, vol. 58, no. 10 p. 1117-1129, D0l:10.1016/j.robot.2010.06.004.

[7] Noureldin, A., Karamat, T.B., Eberts, M.D., El-Shafie, A. (2009). Performance enhancement of MEMS-based INS/ GPS integration for low-cost navigation applications. IEEE Transactions on Vehicular Technology, vol. 58, no. 3, p. 10771096, D0I:10.1109/TVT.2008.926076.

[8] Readdy, G.S., Saraswat, V.K. (2013). Advanced navigation system for aircraft applications. Defence Science Journal, vol. 63, no. 2, p. 131-137, Dol:10.14429/dsj.63.4254.

[9] Sokolovic, V., Dikic, G., Stancic, R. (2014). Adaptive error damping in the vertical channel of the INS/GPS/Baro-altimeter 
integrated navigation system. Scientific Technical Review, vol. 64, no. 2, p. 14-20.

[10] Madgwick, S.O.H., Vaidyanathan, R., Harrison, A.J.L. (2010). An Efficient Orientation Filter for IMU and MARG Sensor Arrays. Report, University of Bristol, Department of Mechanical Engineering, Bristol.

[11] Sokolovic, V., Dikic, G., Stancic, R. (2013). Integration of INS, GPS, magnetometer and barometer for improving accuracy navigation of the vehicle. Defence Science Journal, vol. 65, no. 5, p. 451-455, DOl:10.14429/dsj.63.4534.

[12] Hao, Y., Zhang, Z., Xia, Q. (2010). Research on data fusion for SINS / GPS / magnetometer integrated navigation based on modified CDKF. IEEE International Conference in Progress in Informatics and Computing, p. 1215-1219.
[13] Zhao, H., Wang, Z. (2012). Motion measurement using inertial sensors, ultrasonic sensors, and magnetometers with extended Kalman filter for data fusion. Sensors Journal, vol. 12, no. 5, p. 943-953, D0l:10.1109/JSEN.2011.2166066.

[14] Han, S., Wang, J. (2011). A novel method to integrate IMU and magnetometers in attitude and heading reference systems. Journal of Navigation, vol. 64, no. 4, p. 727-738, D0I:10.1017/ S0373463311000233.

[15] Ahmed, M., Cuk, D. (2005). Strapdown attitude algorithms using quaternion matrix and random inputs. Scientific Technical Review, vol. 55, no. 1, p. 3-13.

[16] Kuipers, J.B. (1999). Quaternions and Rotation Sequences: A Primer with Applications to Orbits, Aerospace and Virtual Reality, Princeton University Press, Princeton. 